\title{
Beam based measurement of beam position monitor electrode gains
}

\author{
D. L. Rubin,* M. Billing, R. Meller, M. Palmer, M. Rendina, N. Rider, D. Sagan, J. Shanks, and C. Strohman \\ Laboratory of Elementary Particle Physics, Cornell University, Ithaca, New York 14853, USA
}

(Received 27 April 2010; published 9 September 2010)

\begin{abstract}
Low emittance tuning at the Cornell Electron Storage Ring (CESR) test accelerator depends on precision measurement of vertical dispersion and transverse coupling. The CESR beam position monitors (BPMs) consist of four button electrodes, instrumented with electronics that allow acquisition of turn-byturn data. The response to the beam will vary among the four electrodes due to differences in electronic gain and/or misalignment. This variation in the response of the BPM electrodes will couple real horizontal offset to apparent vertical position, and introduce spurious measurements of coupling and vertical dispersion. To alleviate this systematic effect, a beam based technique to measure the relative response of the four electrodes has been developed. With typical CESR parameters, simulations show that turn-byturn BPM data can be used to determine electrode gains to within $\sim 0.1 \%$.
\end{abstract}

DOI: 10.1103/PhysRevSTAB.13.092802

PACS numbers: 29.20.db, 29.90.+r

\section{INTRODUCTION}

The Cornell Electron Storage Ring (CESR) test accelerator (CesrTA) [1] beam position monitors (BPMs) have been instrumented with precision turn-by-turn, bunch-bybunch electronics [2]. The system provides the capability of measuring the residual coupling and vertical dispersion that contribute to dilution of the vertical emittance. System tests indicate that the intrinsic position resolution is $<5 \mu \mathrm{m}$. We have developed a beam based technique that provides a measure of the relative gain of the four button electrodes with a precision of about $0.1 \%$.

The strategy we describe for measuring the relative button gain depends on the fact that, in a four electrode BPM, the position of the bunch is overdetermined. By measuring the electrode signal at a number of different beam positions, the relative gains of the electrodes can be calculated. Our approach is similar to the technique of Satoh [3] et al. with some important differences. The method of Satoh depends on knowledge of the response function $F_{i}(x, y)$ that gives the intensity on the $i^{\text {th }}$ button in terms of the position $(x, y)$ of the bunch. The number of fit parameters in the Satoh method is $3 m+3$, where $m$ is the number of measurements. Besides the three button gains, for each measurement there are three unknowns to be fitted: the $x, y$ position and a scaling factor that is proportional to the bunch charge. Each measurement yields four data points, namely, the intensity on each button. As long as $m>4$, the system is overconstrained and the best fit gains can be computed.

As opposed to Satoh, the technique described in this paper does not require knowledge of the response function and only requires that the geometry of the four BPM electrodes be mirror symmetric. With this technique there

*drubin@physics.cornell.edu are just four unknown fitting parameters: three button gains and a geometry scaling factor.

\section{CONSTRAINTS ON THE BUTTON SIGNALS}

The geometry and labeling are as in Fig. 1. The four button electrodes have mirror symmetry and are labeled \#1 through \#4 with buttons \#1 and \#4, along with buttons \#2 and \#3, being diagonally opposite each other. The button signal $B_{i}$ of the $i^{\text {th }}$ electrode of a given BPM will be a function of the position of the beam. For button \#1 write

$$
B_{1}=k f(x, y),
$$

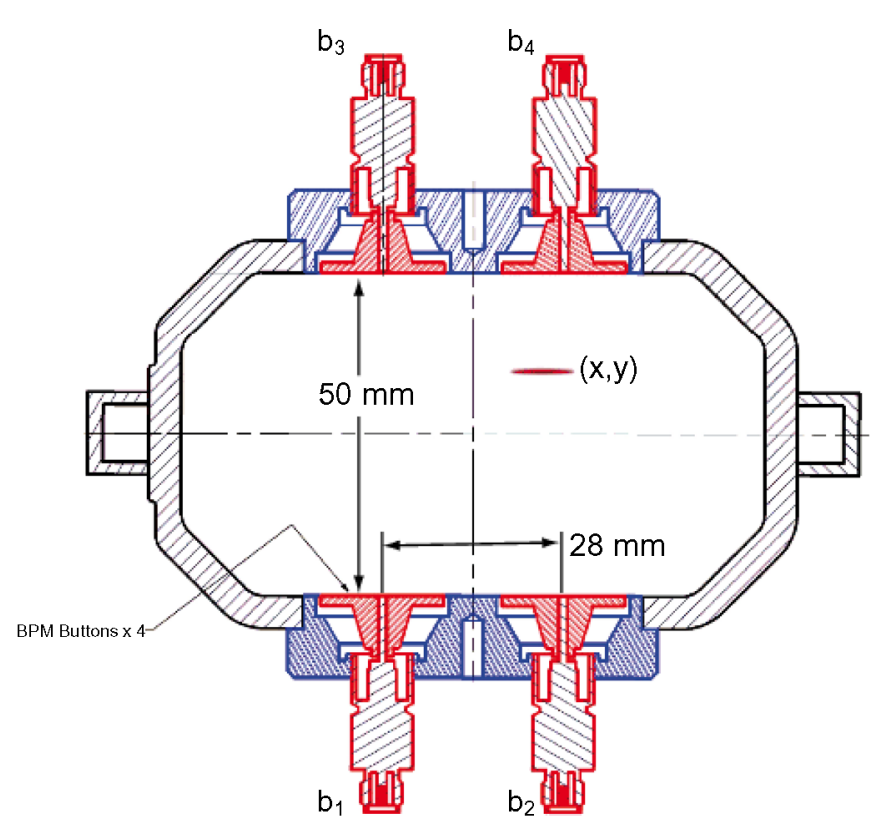

FIG. 1. CESR beam position monitor. Note labeling of buttons $b_{1} \ldots b_{4}$. 
where $(x, y)$ is the beam position at the BPM and $k$ is a constant that depends upon the bunch charge. The beam is assumed to be near the center of the beam pipe so $x$ and $y$ are small compared to the distance from the center of the BPM to the electrodes. In this case, the normalized response function $f$ can be expanded in a double Taylor series. To second order this gives

$$
B_{1} \approx k\left(c_{0}+c_{1} x+c_{2} y+c_{3} x^{2}+c_{4} y^{2}+c_{5} x y\right) .
$$

It is assumed that there is mirror symmetry so that the response functions of the other three buttons can be immediately written as

$$
\begin{aligned}
B_{2} & =k f(-x, y) \\
& \approx k\left(c_{0}-c_{1} x+c_{2} y+c_{3} x^{2}+c_{4} y^{2}-c_{5} x y\right) \\
B_{3} & =k f(x,-y) \\
& \approx k\left(c_{0}+c_{1} x-c_{2} y+c_{3} x^{2}+c_{4} y^{2}-c_{5} x y\right) \\
B_{4} & =k f(-x,-y) \\
& \approx k\left(c_{0}-c_{1} x-c_{2} y+c_{3} x^{2}+c_{4} y^{2}+c_{5} x y\right) .
\end{aligned}
$$

Taking sums and differences of Eqs. (2)-(5) gives

$$
\begin{gathered}
B_{+--+} \equiv B_{1}-B_{2}-B_{3}+B_{4}=4 k c_{5} x y \\
B_{+-+-} \equiv B_{1}-B_{2}+B_{3}-B_{4}=4 k c_{1} x \\
B_{++--} \equiv B_{1}+B_{2}-B_{3}-B_{4}=4 k c_{2} y .
\end{gathered}
$$

Combining the above three equations to eliminate $x$ and $y$ gives an expression that simply relates the button signals:

$$
B_{+--+}=\frac{c_{5}}{k c_{1} c_{2}} B_{+-+-} B_{++--},
$$

where $c_{1}, c_{2}$, and $c_{5}$ are constants that characterize the BPM geometry. To the extent that $B_{+--+}$is proportional to the product $B_{+-+-} B_{++--}$, the second order approximation is valid. (Note that if nonlinear terms are neglected entirely the term $c_{5}=\partial^{2} f / \partial x \partial y=0$ and $B_{+--+}$is zero for all trajectories.)

The relevance of the nonlinearities can be explored with simulations. A finite element code was used to create a map of exact button response as a function of beam position [4]. Figure 2 shows the computed signal for button 1 for each of 209 beam positions. The positions are taken from a $18 \mathrm{~mm} \times 10 \mathrm{~mm}$ grid with the center coinciding with the center of the BPM, and a grid spacing of $1 \mathrm{~mm}$.

The intensities at a single button range from 0.7 to 2.2 on an arbitrary scale as shown in Fig. 2. Insofar as $B_{+--+} \neq$

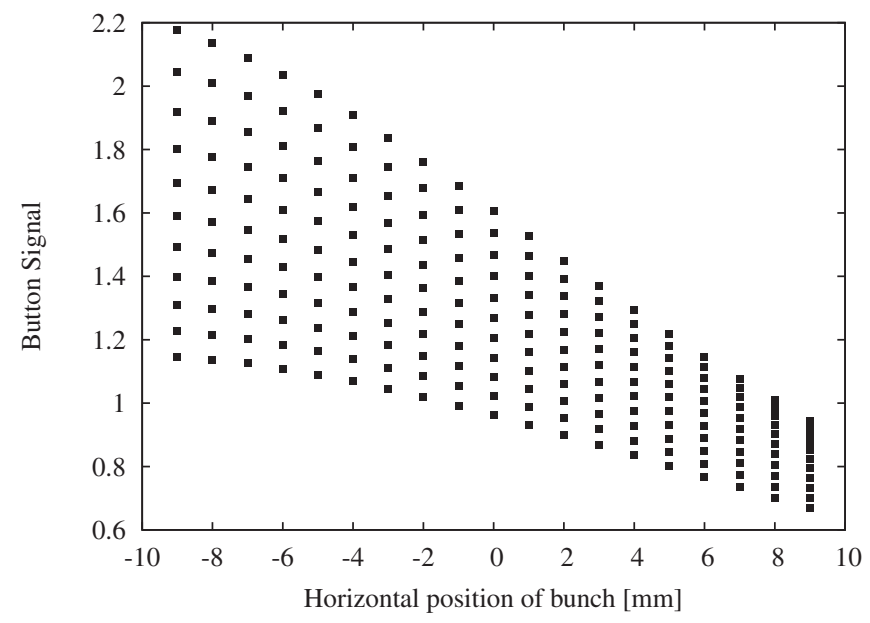

FIG. 2. Button signals from 209 orbits on a $18 \mathrm{~mm} \times 10 \mathrm{~mm}$ grid.

0 , nonlinearities (second derivatives of the response function) are clearly important. We plot $B_{+--+}$as a function of the distance to the center of the BPM in Fig. 3. It is apparent that nonlinearities are significant at distances as small as a couple of $\mathrm{mm}$.

The product $B_{+-+-} B_{++--}$is plotted versus $B_{+--+}$, for the BPM button data generated from the nonlinear map in Fig. 4. We see that our second order approximation is excellent, and that the form of Eq. (9) fits the data with small deviations appearing only at the largest amplitudes.

The above formulation is general in the sense that it depends only on the mirror symmetry of the placement of the four pickup electrodes and is independent of other details of the geometry. The expansion parameter for the Taylor series is $\epsilon=r / r_{0}$, where $r$ is the distance of the beam from the center of the BPM and $r_{0}$ is the distance from the pickup electrodes to the center of the beam pipe. For a typical CESR BPM, $r_{0}=28 \mathrm{~mm}$. As you will see

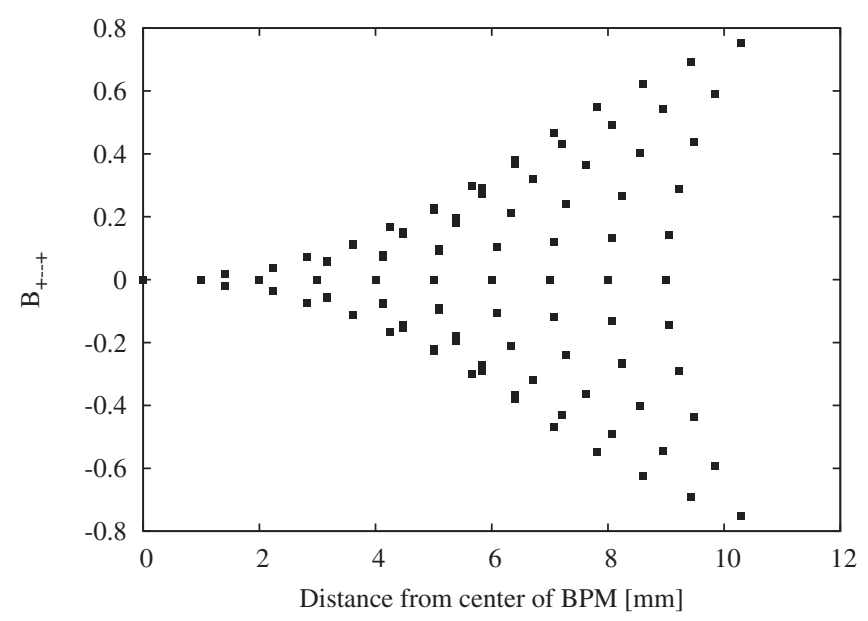

FIG. 3. $B_{+--+}$versus distance $\left(\sqrt{x^{2}+y^{2}}\right)$ from the center of the BPM. 


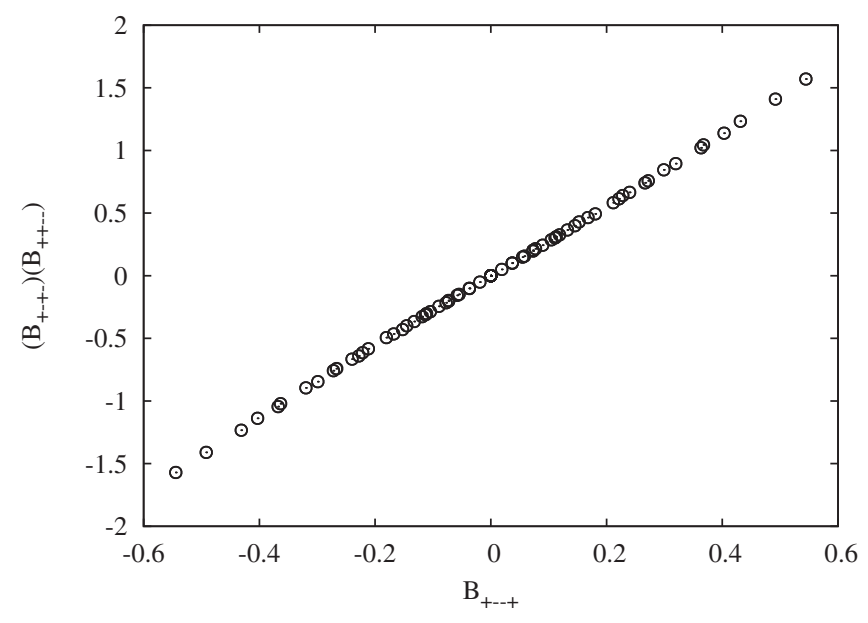

FIG. 4. $B_{+-+-} B_{++--}$vs $B_{+--+}$for points on a $18 \mathrm{~mm}$ by $10 \mathrm{~mm}$ grid with button intensity computed with the map of button signal vs beam position. Deviation from the superimposed straight line, barely evident at even the largest amplitudes, shows the extent to which the higher than second order terms can be ignored. The units are arbitrary.

below, button values corresponding to beam position $r \leq$ $5 \mathrm{~mm}$ are used to determine button gains.

\section{BPM GAIN ERRORS}

If the button electrodes do not all have the same gain, then the relationship between combinations of button intensities defined in Eq. (9) will fail. We explore the effect of gain errors using the previous simulation by reducing the signal on button 4 by $5 \%$. Figure 5 shows a plot of $B_{+-+-} B_{++--}$vs $B_{+--+}$for the simulated grid data. The data is no longer single valued and linear and it is offset from zero.

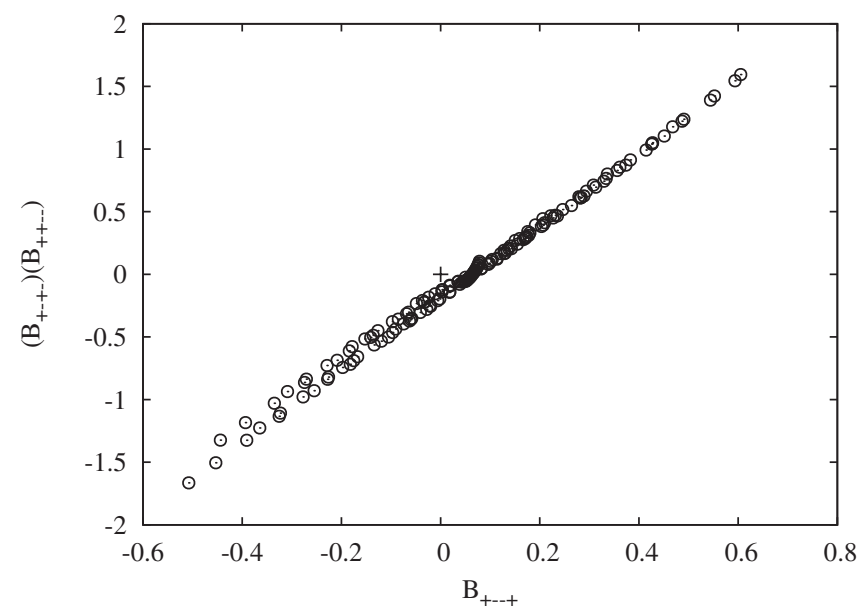

FIG. 5. $B_{+-+-} B_{++--}$vs $B_{+--+}$for points on a $18 \mathrm{~mm}$ by $10 \mathrm{~mm}$ grid with button intensity computed with the nonlinear map. The button 4 signal is reduced by $5 \%$ with respect to buttons 1,2 , and 3 . [The gains $(1: 4)=1,1,1,0.95$.] The + indicates the coordinate $(0,0)$.

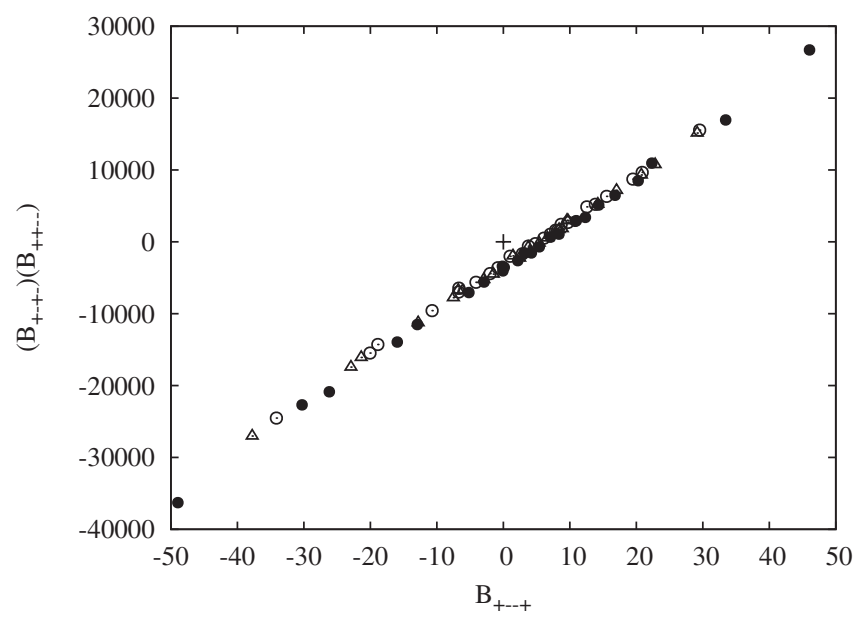

FIG. 6. $B_{+-+-} B_{++--}$vs $B_{+--+}$for measurements of CESR BPM (\#21E). There are 75 measurements distributed on a grid. The + indicates the coordinate $(0,0)$.

\section{MEASUREMENTS}

Now we consider measured data. We expect that for all BPMs of the same type that the product $B_{+-+-} B_{++--}$ will depend linearly on $B_{+--+}$with slope characteristic of that BPM type and with zero intercept. Data for BPM (\#21E) is shown in Fig. 6. The button response is measured for 75 distinct closed orbits arrayed more or less uniformly over a $10 \mathrm{~mm} \times 10 \mathrm{~mm}$ grid. The relationship between $B_{+-+-} B_{++--}$and $B_{+--+}$is very nearly linear, but it is offset from zero in violation of Eq. (9). The offset indicates a variation in the effective gains of the four buttons, either electrical or geometric. Note that each closed orbit data point shown in Fig. 6 is an average of 1024 turns.

As an alternative to using closed orbits, it is possible at CesrTA to take turn-by-turn data while resonantly exciting the beam at the horizontal and vertical normal mode tunes. Over the course of 1024 turns, each BPM will measure

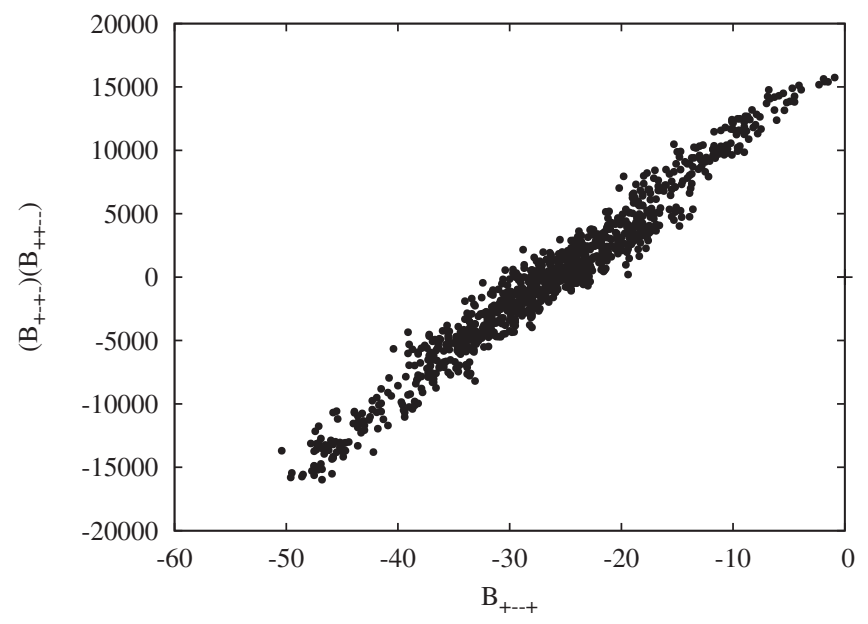

FIG. 7. $B_{+-+-} B_{++--}$vs $B_{+--+}$for measurements of CESR BPM 33. There are 1024 turn-by-turn measurements. 
1024 different positions and the 1024 corresponding quartets of button signals. This method has the advantage that data can be taken very rapidly. The drawback is that the signals cannot be averaged over multiple turns and, hence, the noise will be greater. Resonantly excited turn-by-turn data for BPM (\#33E) is shown in Fig. 7. Again we find that the data is well represented by the fundamental relationship [Eq. (9)] but with a substantial offset from zero. The width of the line is due to the intrinsic noise of the system.

\section{GAIN FIT}

If the deviations from Eq. (9) of the $B_{+-+-} B_{++--}$vs $B_{+--+}$curve are due to gain variations between different button electrodes, a nonlinear least squares fit can be used to determine the button gains $g_{i}$. To do this, the merit function to be minimized is

$$
\begin{aligned}
\chi^{2}= & \sum_{i}^{n}\left(\left(g_{1} B_{1}^{i}-g_{2} B_{2}^{i}-g_{3} B_{3}^{i}+g_{4} B_{4}^{i}\right)\right. \\
& -\frac{c}{I}\left(g_{1} B_{1}^{i}-g_{2} B_{2}^{i}+g_{4} B_{3}^{i}-g_{4} B_{4}^{i}\right) \\
& \left.\times\left(g_{1} B_{1}^{i}+g_{2} B_{2}^{i}-g_{3} B_{3}^{i}-g_{4} B_{4}^{i}\right)\right)^{2},
\end{aligned}
$$

where $c$ is a geometrical coefficient that is common to all BPMs with the same geometry.

The $\chi^{2}$ defined above has a minimum for the best fit gains $g_{i}$ and constant $c$. But there is also a minimum when all the gains are zero. To prevent this, an additional constraint can be imposed by simply setting one of the gains to unity. In practice, in order to limit any systematic effect from our arbitrary selection of a particular button for normalization, we fit the same data four times, normalizing with respect to each of the four buttons, and then average the results.

\section{SIMULATION}

We use simulated data to test the validity of the algorithm. We introduce gains that in general are different for each button, and then use the fitting algorithm to determine those gains. We generate button data corresponding to beam positions on a rectangular grid. The quality of the fit will depend on the size of the grid in $x-y$ space as well as the measurement error of the individual button signals. If the grid is too small, then the fit will be sensitive to measurement errors. However, the fit algorithm depends on the assumption that $r^{2} / r_{0}^{2} \ll 1$, so if the grid is too large, the algorithm will fail.

Figure 8 shows the results of the fits to simulated button data for three different grid sizes, $\pm 3 \mathrm{~mm}$ in both $x$ and $y$, $\pm 5 \mathrm{~mm}$ and $\pm 10 \mathrm{~mm}$, and for button measurement resolutions ranging from $0.1 \%$ to $1 \%$ of the average button signal value. Each fit is based on 1000 measurements, and for each fit we compute the difference of the fitted gains from the real gains $\left(\Delta g_{j}^{i}=g_{\text {fitted }, j}^{i}-g_{\text {real }, j}^{i}\right)$. The procedure

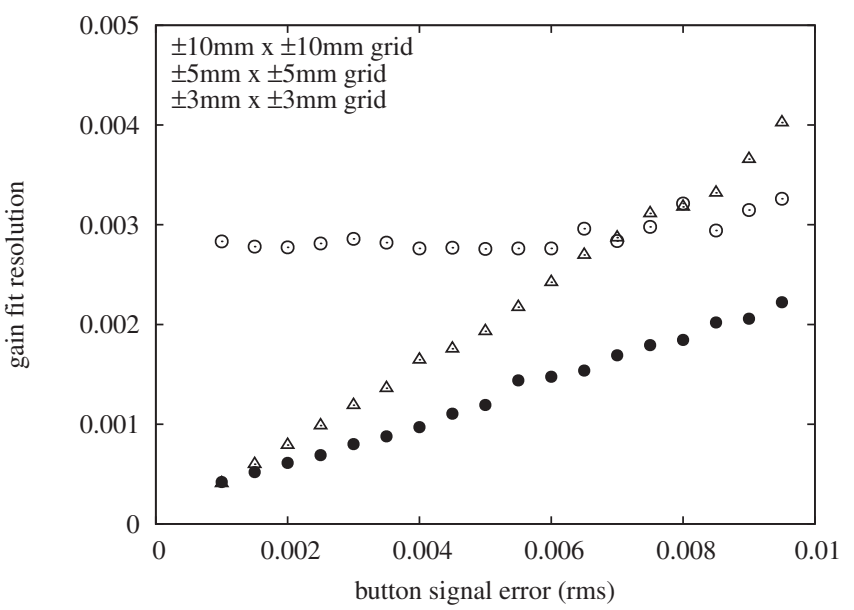

FIG. 8. Simulation of the resolution of the fitted gain as a function of the BPM button measurement resolution (button signal error) for three different size grids. The $\pm 5 \mathrm{~mm}$ square grid is characteristic of resonantly excited turn-by-turn data. The model is good to second order in grid size. Third order effects are evident in the limited resolution of the fits to the data in the $\pm 10 \mathrm{~mm}$ square grid.

is repeated 1000 times, each time with a different seeding of Gaussian measurement errors. Finally, we compute, and plot, the root mean square of the difference,

$$
\sigma_{j}=\frac{1}{N_{\text {seeds }}} \sum_{i}\left(\Delta g_{j}^{i}\right)^{2} .
$$

We expect the button resolution for a single turn to be of order $0.2 \%$. Then according to the simulation we can determine the gains to within $0.1 \%$ with a $\pm 5 \mathrm{~mm}$ by $\pm 5 \mathrm{~mm}$ sampling region.

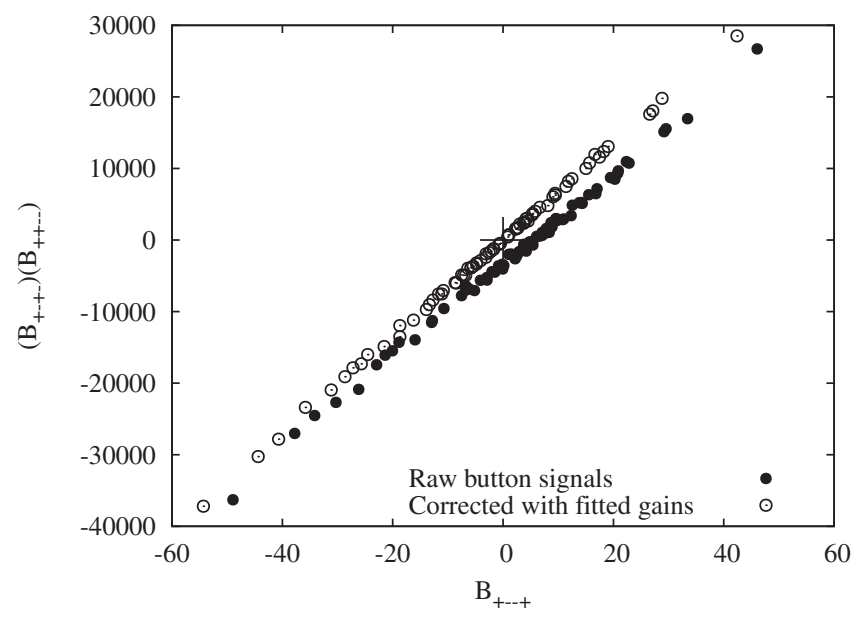

FIG. 9. $B_{+-+-} B_{++--}$vs $B_{+--+}$for CESR BPM (\#21E). There are 75 measurements distributed on a grid. The filled circles are the raw data. The open circles are the data corrected with the fitted gains. The fitted gains are $g(1: 4)=0.993$, $0.999,1.020,0.988$. The + indicates the coordinate $(0,0)$. Note that the fit eliminates the offset from zero. 


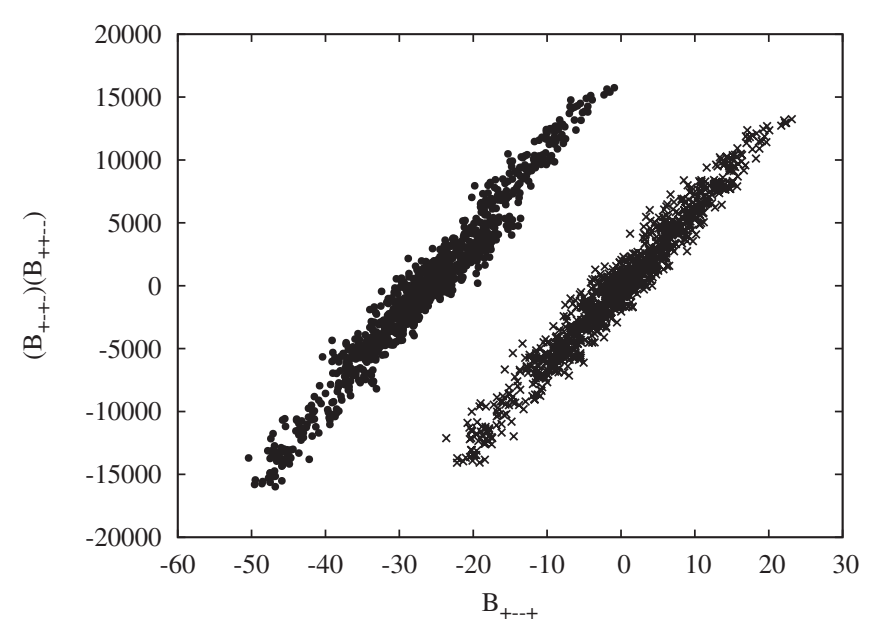

FIG. 10. $B_{+-+-} B_{++--}$vs $B_{+-+-}$for a turn-by-turn measurement at BPM 66. There are 1000 turns of position measurements. The filled circles are derived from the measured button intensities. The $x$ points are based on button values corrected with the fitted gains. The fitted gains are $g(1: 4)=1.001,0.982$, $0.973,1.044$.

\section{FITTING ORBIT DATA}

As a test of the method, a fit to orbit data for BPM $(\# 21 \mathrm{E})$ is shown in Fig. 9. For each of three sets of data we measure button intensities for 25 closed orbits on a grid that is approximately $\pm 9 \mathrm{~mm}$ by $\pm 5 \mathrm{~mm}$. The three sets of data are fit independently. Agreement between the fitted gains for the three data sets is reasonably good.

\section{TURN-BY-TURN DATA}

The beam is resonantly excited at the horizontal and vertical normal mode frequencies. The amplitude of the transverse oscillations is $\sim \pm 5 \mathrm{~mm}$. We collect $\mathrm{BPM}$

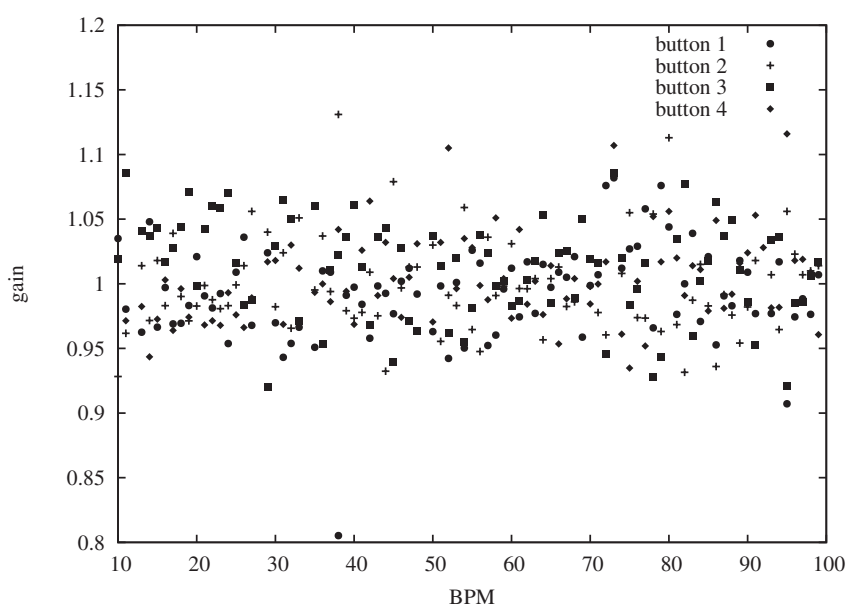

FIG. 11. Fitted gains from a turn-by-turn data set for all 100 beam position monitors, normalized so that the average of the four button gains for each BPM is unity.

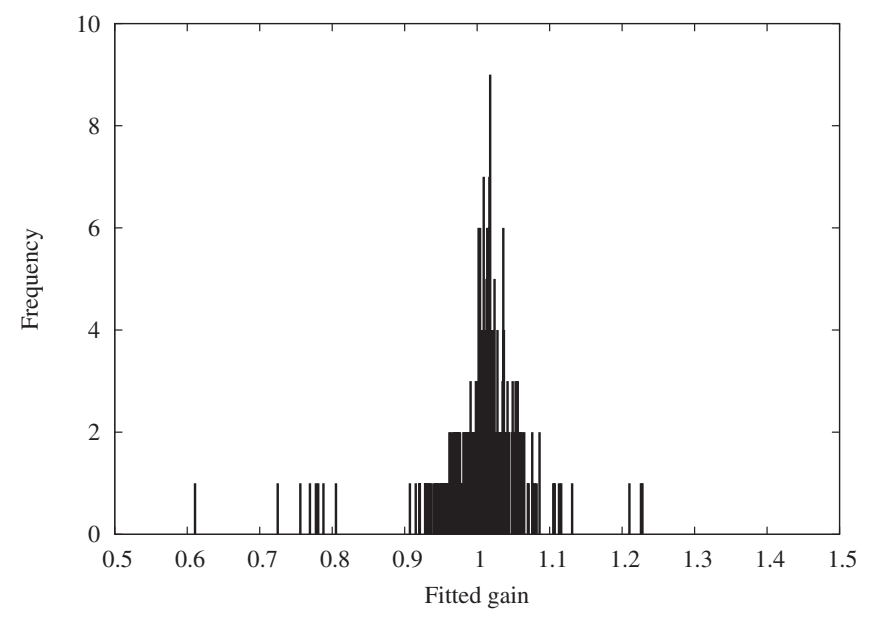

FIG. 12. Distribution of fitted gains for the data plotted in Fig. 11.

button data on each of 1024 turns simultaneously for the 100 beam position monitors in CESR. An example of fitted data at one BPM is shown in Fig. 10. Gains computed for 100 BPMs based on turn-by-turn data are shown in Fig. 11. The distribution of fitted gains for the turn-by-turn data is shown in Fig. 12.

\section{TURN-BY-TURN VS CLOSED ORBIT DATA}

The technique is evidently effective with either turn-byturn or closed orbit data. However, the procedure for collecting turn-by-turn data is much more efficient. A robust fit requires that the BPM be sampled uniformly over a $\pm 5 \mathrm{~mm} \times \pm 5 \mathrm{~mm}$ grid. That is readily accomplished in turn-by-turn data taking mode for all BPMs simultaneously by resonant excitation of the beam at the horizontal and vertical tunes. In closed orbit mode, complete sampling depends on generating closed bumps to manipulate the orbit in each BPM. In CESR there are insufficient dipole correctors to adequately sample all of the BPMs with closed orbits simultaneously. The advantage of closed orbit data, in that each point is the average of 1024 single turn measurements, is balanced by the simplicity of collecting a very large number of data points in the turn-by-turn mode.

\section{CONCLUSION}

We have derived a relationship among the intensities of the four buttons of a beam position monitor with horizontal and vertical mirror symmetry. We show how the relationship can be used to make a beam based measurement of the relative gains of the four buttons. A simulation study indicates that, if the resolution of the measurement of the intensity of a single button is $\sim 0.2 \%$, the gains can be determined with a precision of $0.1 \%$. We have used turnby-turn data to fit the gain for all 100 CESR beam position 
monitors. The standard deviation of the distribution of measured gains is about $6 \%$, consistent with the specifications of the system electronics. This turn-by-turn calibration of button gains will be implemented as part of the standard initialization of measurements of dispersion and transverse coupling.

\section{ACKNOWLEDGMENTS}

We would like to thank the CESR machine group for maintaining and operating the CESR test accelerator, thus enabling the measurements necessary to develop the gain fitting technique. This work was supported by the National Science Foundation and the U.S. Department of Energy under Contracts No. PHY-0734867 and No. DE-FC0208ER41538.

[1] M. Palmer et al., in Proceedings of the 23rd Particle Accelerator Conference, Vancouver, Canada, 2009 (IEEE, Piscataway, NJ, 2009).

[2] M. Palmer et al., Proceedings of the 2010 International Particle Accelerator Conference, p. 1191.

[3] K. Satoh and M. Tejima, in Proceedings of the Particle Accelerator Conference, Dallas, TX, 1995 (IEEE, New York, 1995), p. 2482.

[4] R. Helms and G. Hoffstaetter, Phys. Rev. ST Accel. Beams 8, 062802 (2005). 